\title{
Viability study of a multiplex diagnostic platform for Chagas disease
}

\author{
Leonardo Foti', Bruna de Paula Fonseca e Fonseca ${ }^{2}$, Lilian Dias Nascimento², \\ Christiane de Fatima Silva Marques ${ }^{2}$, Edmilson Domingos da Silva ${ }^{2}$, Cesar Augusto Barros Duarte ${ }^{1}$, \\ Christian M Probst' ${ }^{1}$, Samuel Goldenberg1, Antônio Gomes Pinto², Marco Aurélio Krieger ${ }^{1++}$
}

${ }^{1}$ Instituto Carlos Chagas-Fiocruz, R. Prof. Algacyr Munhoz Mader 3775, 81350-010 Curitiba, PR, Brasil 2Laboratório de Tecnologia Diagnóstica, Biomanguinhos-Fiocruz, Rio de Janeiro, RJ, Brasil

\begin{abstract}
A new multiplex assay platform was evaluated to detect Trypanosoma cruzi infection using the recombinant antigens CRA, FRA, CRAFRA fusion and parasite lysate. The antigens presented different sensitivity and specificity in a singleplex test when compared to a serial dilution of two pools comprising 10 positive serum samples and one pool of 10 negative samples. The recombinant protein CRA presented lower sensitivity (55\%) in contrast to the 100\% specificity and sensitivity of FRA, CRAFRA and T. cruzi lysate. These antigens also showed good results in a duplex test and the duplex test with CRAFRA/T. cruzi lysate showed better performance with 100\% specificity and sensitivity, as well as a lower cut-off value in comparison to the other duplex test, FRA/T. cruzi lysate. Hence, when the antigens were used in duplex format, both tests showed decreased cut-off values and no interference between different bead sets, resulting in increasing sensitivity and specificity. The results of these multiplex tests show that they could be an alternative to singleplex detection for Chagas disease, and also indicate the necessity of using multiplex diagnostic tools to increase the sensitivity and specificity for diagnostic tests. Emerging data from the T. cruzi genome and from its ORFeome project will also allow the identification of new antigens for this disease detection application.
\end{abstract}

Key words: Chagas disease - diagnostic - multiplex - bead-based assay - Trypanosoma cruzi

Over one billion people worldwide (1/6 of the world's population) suffer from one or more neglected diseases (O'Conell 2007). Beyond mortality, neglected diseases cause severe and permanent disabilities and deformities, hampering human development and resulting in significant socio-economic losses (King et al. 2006, Hopkins et al. 2007).

Chagas disease is one of the world's leading causes of heart disease, representing the second highest illness burden among tropical diseases in the Americas (TDR 2007). The protozoan Trypanosoma cruzi is the etiologic agent of the disease in mammals; the parasite is transmitted by blood-sucking triatomine bugs, blood transfusion or across the placenta (Chagas 1909, Brener et al. 2000).

Since 1990, a series of multinational initiatives based on vector control, systematic screening of blood donors in all endemic countries and detection and treatment of congenital transmission have been launched for control and elimination of Chagas disease. These strategies have led to significant reductions in the number of infected people worldwide. However, approximately eight million people are still infected with $T$. cruzi; thus Chagas disease continues to represent a health threat for an estimated 28 million people (TDR 2007).

+ Corresponding author: mkrieger@fiocruz.br

Received 19 May 2009

Accepted 16 June 2009
Accurate and early diagnosis is essential for correct therapeutic treatment of $T$. cruzi infected patients. However, Chagas disease is often not diagnosed sufficiently early in most asymptomatic or oligosymptomatic infected individuals (MSF 2008). In several cases, by the time patients presented clinical manifestations of advanced chronic Chagas disease, specific treatment with currently available drugs had limited effectiveness (Coura 2007). T. cruzi diagnosis is frequently reached after multiple serological tests, in combination with epidemiological data and clinical symptoms (Cooley et al. 2008). Thus, mechanisms for epidemiologic surveillance and accurate diagnosis are necessary to compensate for the lack of inexpensive and effective diagnostic tools and therapies that meet the requirements of health needs in endemic countries (Burgess et al. 2006).

Chagas diagnosis relies on different techniques according to the phase of the disease. Direct techniques to detect the presence of the parasite are used mainly in the acute phase when the parasite is present in high amounts in the blood of the infected host. On the other hand, due to low parasitaemia, chronic phase diagnosis is based on indirect techniques that assay for specific immune responses against $T$. cruzi antigens. Several enzyme-linked immunosorbent assays (ELISAs) are commercially available and are the main tests currently used for serological diagnosis of Chagas disease. However, as false positive results are possible, other techniques such as indirect haemagglutination assay (IHA) and indirect immunofluorescence assay (IFA) are recommended for confirmation. In fact, even if a test with very high sensitivity is used, a second assay is usually recommended since different assays detect antibodies of different specificities (Lopez-Antuñado 1997). 
Despite this recommendation, there is still a great degree of discordance among results obtained in different laboratories (Schumunis \& Cruz 2005). These tests also have other important drawbacks, including low processing speed, high labour time, low throughput capacity, limited multiplex capability and high cost (Joos et al. 2002, Yan et al. 2005). These points and the fact that Chagas disease treatment is monitored on a serodiagnostic basis prompts the use of antigens that allow the detection of specific antibodies in acute and chronic infections (Villa et al. 2007). The use of defined antigens together with parasite lysates in a multiplexed assay would be a significant improvement for serodiagnostic testing and immunopathologic studies of Chagas disease.

In the past decade, several technologies have emerged as diagnostic tools capable of improving diagnosis by using multiplex principles. The diagnostic process becomes faster, less expensive, and the hands-on time in laboratories decreases substantially since these platforms can be fully automated (Jani et al. 2002). One of the most promising multiplex techniques consists of a suspension array created by immobilising capture reagents on the surface of encoded microspheres in conjunction with flow cytometry analysis (Vignali 2000, Jani et al. 2002, Joos et al. 2002). Briefly, serum antibodies are first bound to antigens chemically coupled to the surface of microspheres, and then fluorochrome-conjugated reporter antibodies are bound onto the serum antibodies. As the microspheres pass sequentially through the microfluidic system, fluorescence signals from each microsphere and the reporter antibodies are measured to reveal the identity of bead set and the presence of the captured antibodies, respectively (Joos et al. 2002).

Microsphere-based immunoassays allow quantitative and qualitative analysis of multiple targets with a unique combination of features, including high throughput capacity, small sample volume, high reproducibility, high sensitivity (especially with efficient fluorochromes such as phycoerythrin) and a wide dynamic range (Yan et al. 2005). This system is an open platform for analysis of different molecular interactions and has been used for detection of several molecules, from cytokines (Prabhakar et al. 2002) to single-nucleotide polymorphisms (Lee et al. 2004). It has also been used as a serology tool for screening of antibodies against viruses (Dias et al. 2005) and bacteria (Lal et al. 2004), as well as autoantibodies (Martins et al. 2004).

In this study, we developed a microsphere-based immunoassay for T. cruzi infection diagnosis. By using different types of parasite antigens (crude extracts and recombinant proteins) we describe a multiplex test capable of detecting T. cruzi infection in serum samples from patients, demonstrating that this platform can be used to develop new and more informative diagnostic tests for neglected diseases.

\section{MATERIALS AND METHODS}

Human sera - Negative and positive sera for Chagas disease were obtained from the National Panel for Blood Screening Quality Control (AEQ); sera were elaborated and tested by the Technology Institute for
Immunobiologicals (Bio-Manguinhos/Fiocruz) and the National Institute for Health Quality Control (Fiocruz). All sera samples were analysed using conventional serologic tests (e.g., IFI, IHA and ELISA) to detect specific IgG performed at Bio-Manguinhos. Three serum pools were constructed: one "negative" pool, consisting of 10 sera that were negative in all assays, and two "positive" pools, containing 10 sera each that provided unequivocally positive results on all tests. These samples were also individually used throughout the experiments, with addition of 10 other negative sera.

Antigens - The T. cruzi recombinant antigens CRA and FRA, their recombinant fusion protein designated CRAFRA (Krieger et al. 1992) and T. cruzi lysate (Leon et al. 2001) were produced by Bio-Manguinhos/FIOCRUZ.

Microsphere coupling to T. cruzi antigens - Coupling of recombinant antigens or T. cruzi lysate to paramagnetic carboxylated microspheres (Luminex Corp, Austin-TX, USA) was performed according to the manufacturer's instruction. Briefly, $2 \times 10^{6}$ microspheres were vortexed and sonicated to assure homogeneous distribution. The microsphere suspension was then washed twice with double-distilled water $\left(\mathrm{dH}_{2} \mathrm{O}\right)$ and suspended in 80 $\mu \mathrm{L}$ of activation buffer $(100 \mathrm{mM}$ sodium phosphate, $\mathrm{pH}$ 6.2). Solutions (10 $\mu \mathrm{L}$ of each) of N-hydroxysulfosuccinimide (Pierce, Rockford-IL, USA) and 1-ethyl-3(3-dimethylaminopropyl)-carbodiimide hydrochloride (Pierce), both diluted to $50 \mathrm{mg} / \mathrm{mL}$ in $\mathrm{dH}_{2} \mathrm{O}$, were added to stabilise the reaction and activate the microspheres. After mixing, the microspheres were incubated for 20 min in the dark at RT. The activated microspheres were subsequently washed with coupling buffer (50 mM MES, pH 5.0 and PBS 1 x, pH 7.0), after which $200 \mu \mathrm{L}$ of antigen solution was added. Microspheres were incubated with rotation for $2 \mathrm{~h}$. After incubation, the microspheres were washed with washing buffer (PBS, containing 1\% bovine serum albumin (BSA), $0.05 \%$ Tween 20 ) and suspended in $500 \mu \mathrm{L}$ of blocking/storage buffer (PBS containing $1 \% \mathrm{BSA}$ and $0.02 \%$ sodium azide). The microspheres were counted with a haemocytometer, adjusted to a concentration of $4 \times 10^{4}$ microspheres $/ \mathrm{mL}$ with storage buffer and stored protected from light at $2-8^{\circ} \mathrm{C}$.

Microsphere immunoassay standard protocol - All incubations were performed at $37^{\circ} \mathrm{C}$ on a microplate shaker (set at $600 \mathrm{rpm}$ ). For the wash steps, a Hydroflex plate washer with a magnetic plate support (TECAN, Durham, NC, USA) was used. Serum samples were diluted in assay buffer (PBS containing 1\% BSA, $0.05 \%$ Tween 20) and the assay was conducted using standard procedures described by the manufacturer (Luminex Corp, Austin-TX, USA). A total of $50 \mu \mathrm{L}$ containing approximately 2,000 coupled paramagnetic microspheres was added to each well of a 96-well plate. For multiplex assays, the same number of coupled microspheres was added to each well for each bead set. Diluted serum (50 $\mu \mathrm{L})$ and microspheres were mixed and incubated for 30 min in the dark. The microspheres were then washed twice with $100 \mu \mathrm{L}$ of wash buffer. Goat anti-human IgG conjugated to phycoerythrin (Sigma) diluted 1:100 
in assay buffer was added and microspheres were incubated for $30 \mathrm{~min}$ in the dark. The microspheres were washed twice with $100 \mu \mathrm{L}$ of wash buffer and reporter fluorescence, expressed as median fluorescence intensity (MFI), was determined with a LabScan 100 device (ONE LAMBDA, USA).

Cut-off determination - Twenty negative serum samples were assayed in duplicate and the obtained MFI was considered valid when the bead count reached a minimum of 100 beads per bead set per well. The net MFI for each serum and antigen in the assay was obtained by subtracting the MFI from background wells (no serum added) and then calculating the mean of the replicates. The cut-off values were determined from the mean MFI of the duplicates added to four times the standard deviation of the net MFI of the negative samples: cut-off = mean of net MFIs + (4 x STD of net MFIs).

Asep_pop determination - This value represents the fold difference between the positive and negative serum population. $\Delta$ sep_pop was calculated as the least positive serum net MFI value minus the highest negative serum net MFI value. The obtained value was divided by the standard deviation of the negative samples $[\Delta$ sep_pop = (least positive net MFI - highest negative net MFI)/ standard deviation of negative samples].

A CRAFRA antigen



Sample dilution

C FRA antigen



\section{RESULTS}

Singleplex assay for Chagas disease diagnosis - Development of a microsphere-based immunoassay for Chagas disease required an initial assessment of the efficiency of antigen coupling to the microspheres and an analysis of the best sample dilution to be used for the assay. For this purpose, we analysed three serum sample pools derived from the AEQ panel: two positive for Chagas disease and one negative.

Each Chagas antigen was conjugated to an individual microsphere set. Serial dilutions of pools ranging from 1:25-1:3200 were made and assayed with the standard assay protocol. All analyses were made singly in order to assess the range of fluorescence generated by each individual microsphere set.

Despite the different profiles of each dilution curve, a 1:200 dilution gave optimal signal results for all antigens (Fig. 1). This dilution also allowed a greater degree of discrimination between negative and positive samples, thus it was used in all subsequent experiments.

Singleplex specificity analysis - Once the sample dilution was established, experiments were performed to determine whether the assays were indeed measuring antibodies raised against $T$. cruzi; 20 positive and 20 negative sera for Chagas disease were individually assayed against all antigens.

B

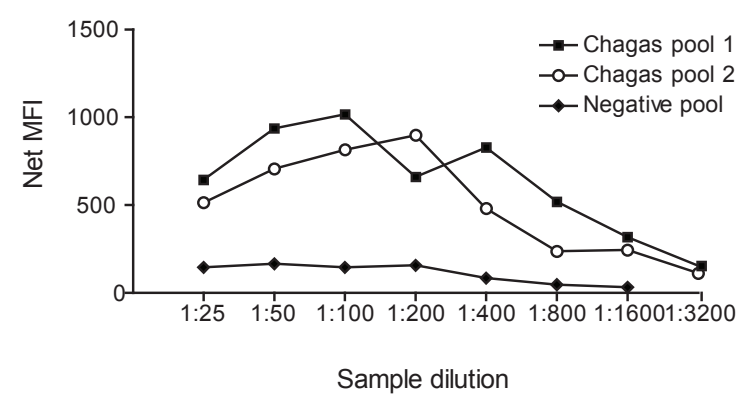

D

T.cruzi lysate antigen

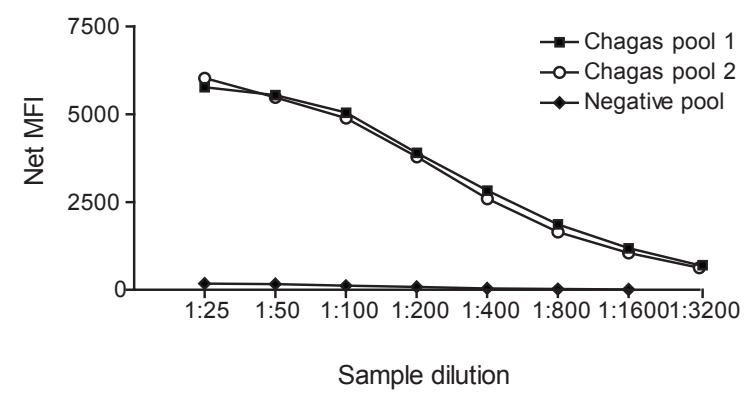

Fig. 1: efficiency of Trypanosoma cruzi antigens coupling to microspheres. Net median fluorescence intensity (MFI) of antigen detection in serial dilution samples of Chagas pool 1, Chagas pool 2 and negative pool. Antigens used: CRAFRA (A), CRA (B), FRA (C) T. cruzi lysate (D). 
Fig. 2 demonstrates a clear separation of negative and positive samples for three of the antigens analysed. The $\Delta$ sep_pop presented a 50.7, 21.8 and 15.9-fold difference between positive and negative sera populations for CRAFRA, lysate and FRA antigens, respectively. No false positives were detected for these antigens (100\% specificity) at their respective cut-off lines. The CRA antigen, however, presented a lower sensitivity (55\%) as it was unable to detect all positive samples; this antigen was consequently excluded from all subsequent tests.

Duplex immunoassay - Two duplexes were tested: one containing two microsphere sets each coupled with CRAFRA or lysate antigens and another duplex containing two microsphere sets each coupled with FRA or lysate antigens.

All three antigens in the duplex assays showed comparable results for positive and negative samples when compared to their singleplex performance (Fig. 3). Interference between the different microsphere sets was investigated by comparing the net MFIs generated by the singleplex assay with those generated by the duplex assay. Fig. 3 shows that no interference was observed between bead sets. Intensity dispersion of the samples was also maintained in the duplex assays with the exception of the CRAFRA antigen, which presented slightly higher net MFI signals in the duplex assay format (Fig. 3C).

\section{DISCUSSION}

Diagnostic tools are important for identification of diseases and can determine their correct treatment. However, current diagnostic tools are costly and not sensitive or specific enough to be widely used in public health programs in developing countries. Efficient diagnostic tools for such countries must be able to detect a large number of diseases in an inexpensive, fast and precise way (Urdea et al. 2006, O’Meara et al. 2007). A multiplexing tool that can quickly and accurately identify specific antibodies against a certain pathogen is critically needed for both public health improvement and epidemiological surveillance.

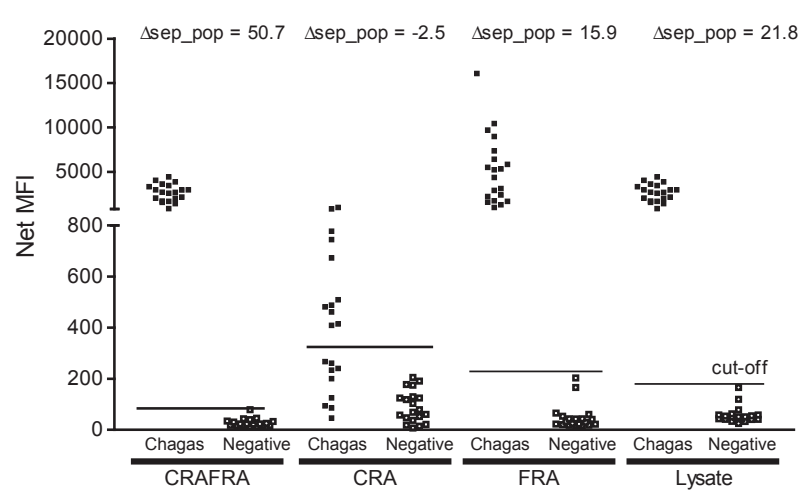

Fig. 2: specificity analysis for Chagas individual antigens. Net median fluorescence intensity (MFI) obtained by the 1:200 dilution of 20 Chagas positive serum samples and 20 negative sera evaluated in singleplex assays against the antigens CRAFRA, CRA, FRA and Trypanosoma cruzi lysate.

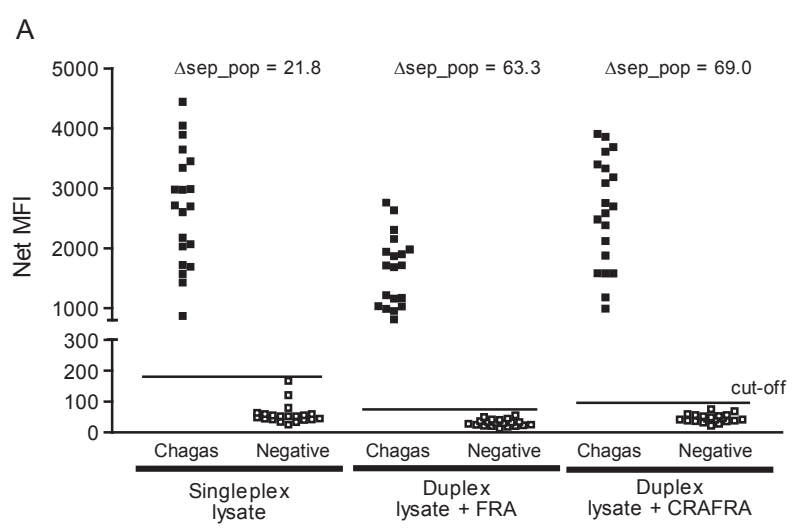

B

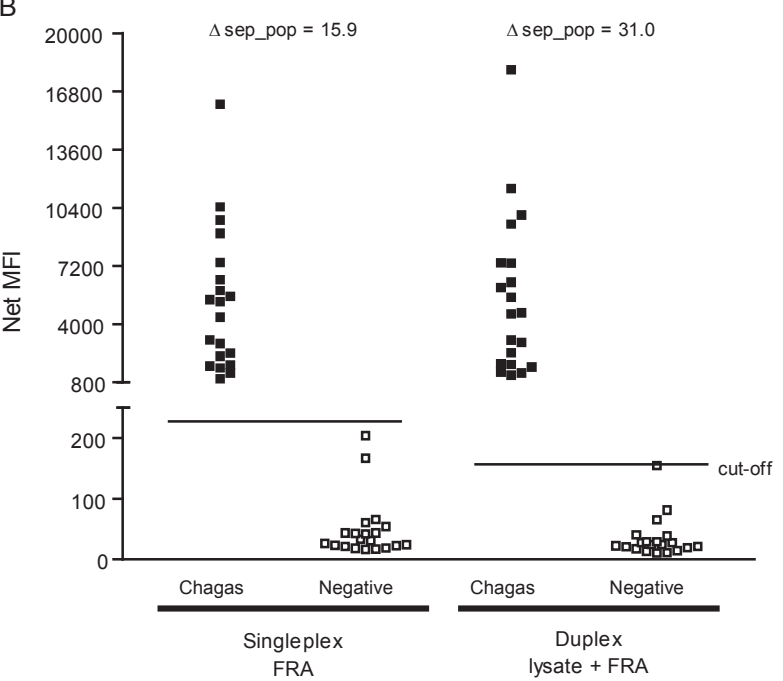

C

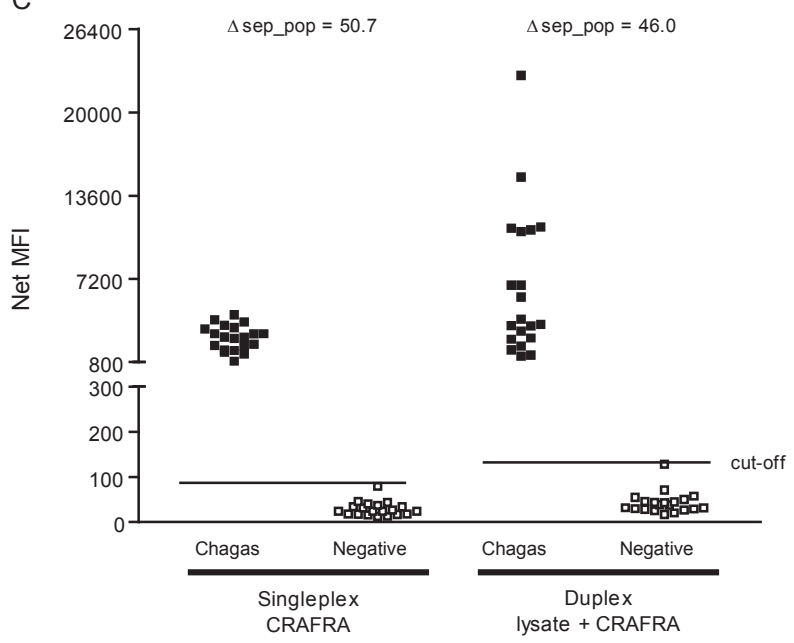

Fig. 3: comparison of the performance of duplex assays relative to singleplex assays. Net median fluorescence intensity (MFI) obtained by the analysis of 1:200 dilutions of Chagas positive and negative sera evaluated in singleplex Trypanosoma cruzi lysate antigen, duplex lysate/FRA and duplex lysate/CRAFRA (A), singleplex FRA and duplex lysate/FRA (B), singleplex CRAFRA and duplex lysate/ CRAFRA (C). 
In this study we developed a microsphere-based assay for Chagas disease using the antigenic properties of CRAFRA and FRA recombinant proteins (Krieger et al. 1992), which are different $T$. cruzi antigens, as well as a T. cruzi lysate (Leon et al. 2001).

As the sensitivity of each antigen affects the resulting output, our first approach was to identify the response obtained by each antigen upon comparison with positive and negative serum pools. All antigens presented high net MFI values for the positive pools and lower net MFI values for the negative pool. However, the CRA antigen showed high reactivity with the negative serum pool and low reactivity with the positive serum pool at the given detection threshold. This result, and the fact that the CRAFRA antigen showed better distinction between positive and negative serum pools, reinforces the importance of combining distinct antigens for better detection.

When the CRAFRA, FRA and lysate antigens were compared with individual samples in singleplex format, they were able to clearly differentiate the negative from the positive sera samples, whereas the CRA antigen failed to do so. This was not an unexpected result since CRA is part of the fusion protein CRAFRA, which presented high MFI values and the lowest cut-off even when compared to the results obtained with FRA alone. This result shows that CRA used together with FRA does not interfere with CRAFRA sensitivity. For this reason, CRA antigen was excluded from the subsequent tests.

In order to evaluate multiplex capability, duplex assays utilising beads coupled with lysate/FRA and lysate/ CRAFRA antigens were performed. Interference between microsphere sets is an important consideration for multiplex assays, but comparison of MFIs generated in the singleplex and duplex assays showed no interference between microsphere sets.

The duplex of lysate/CRAFRA showed the lowest cut-off value and was able to differentiate between negative and positive samples, with only one negative serum sample within the cut-off limit. The lysate/FRA duplex was also able to differentiate between negative and positive samples while maintaining the same profile of sample dispersion, but the same negative sample was detected as a false positive

The duplex assays developed in the present study are sensitive, rapid and show good specificity. Thus, the duplex microsphere assay has the potential to become a viable alternative to standard ELISA, as it requires much smaller sample volumes as well as greatly reduced time and labour spent when assaying a large number of samples. Diagnosis of T. cruzi infection using a multiple antigen strategy is therefore feasible, as multiplex technology can be used to diagnose multiple diseases in one single reaction. Also, it allows the screening of different physiological manifestations of the host during infection evolution.

Although this was a preliminary study, the present work showed robust results, especially in the ability of this test to separate the positive and negative sample populations by the obtained $\Delta$ seq_pop values. The use of a larger sample size with different characteristics such as low positives as well as different disease samples to address cross reactivity is important for realisation of the true capability of this multiplex test as a diagnostic tool.

It is important to note that, after publication of the T. cruzi reference genome (El-Sayed et al. 2005), a wealth of information became available, enabling new approaches for Chagas disease diagnosis. During the search for new markers, all coding sequences identified in the T. cruzi genome were cloned into an appropriate vector, resulting in the construction of a collection named ORFeome (Rual et al. 2004). These coding sequences can be expressed in a high-throughput manner (Luan et al. 2004, Chen et al. 2008) and used for screening patient sera, enabling the potential identification, at unprecedented speed, of new markers for diagnosis of Chagas disease. This strategy could also be used to find more specific markers for clinic subtypes, disease evolution and for the evaluation of Chagas disease treatments. The T. cruzi ORFeome is currently under construction in our laboratory and, in the near future, will be used for the abovementioned screenings, as well as for many other applications in different research fields involving $T$. cruzi biology and pathogenesis. This resource will present an invaluable toolset for researchers working with $T$. cruzi and Chagas Disease.

\section{ACKNOWLEDGEMENTS}

To Alexandre Dias Tavares, from the Institute of Molecular Biology of Paraná, Brazil, for the critical review of the manuscript.

\section{REFERENCES}

Brener Z, Andrade ZA, Barral-Neto M 2000. Trypanossoma cruzi e doença de Chagas. Guanabara Koogan, Rio de Janeiro, 431 pp.

Burgess DCH, Wasserman J, Dahl CA 2006. Global health diagnostics. Nature 444 (Suppl. 1): 1-2.

Chagas C 1909. Nova tripanozomiase humana. Estudos sobre a morfolojía e o ciclo evolutivo de Schizotrypanum cruzi n.gen., n.sp., ajente etiolójico de nova entidade morbida do homen. Mem Inst Oswaldo Cruz 1: 159-218.

Chen Y, Qiu S, Luan CH, Luo M 2008. A high throughput platform for eukaryotic genes. Methods Mol Biol 426: 209-220.

Cooley G, Etheridge RD, Boehlke C, Bundy B, Weatherly DB, Minning T, Haney M, Postan M, Laucella S, Tarleton RL 2008. High throughput selection of effective serodiagnostics for Trypanosoma cruzi infection. PLoS Med 2: e316.

Coura JR 2007. Chagas disease: what is known and what is needed - a background article. Mem Inst Oswaldo Cruz 102 (Suppl. I): 113-122.

Dias D, Van Doren J, Schlottmann S, Kelly S, Puchalski D, Ruiz W, Boerckel P, Kessler J, Antonello JM, Green T, Brown M, Smith J, Chirmule N, Barr E, Jansen KU, Esser MT 2005. Optimization and validation of a multiplexed luminex assay to quantify antibodies to neutralizing epitopes on human papillomaviruses 6,11 , 16 and 18. Clin Diagn Lab Immunol 12: 959-969.

El-Sayed NM, Myler PJ, Bartholomeu DC, Nilsson D, Aggarwal G, Tran AN, Ghedin E, Worthey EA, Delcher AL, Blandin G, Westenberger SJ, Caler E, Cerqueira GC, Branche C, Haas B, Anupama A, Arner E, Aslund L, Attipoe P, Bontempi E, Bringaud F, Burton P, Cadag E, Campbell DA, Carrington M, Crabtree J, 
Darban H, da Silveira JF, de Jong P, Edwards K, Englund PT, Fazelina G, Feldblyum T, Ferella M, Frasch AC, Gull K, Horn D, Hou L, Huang Y, Kindlund E, Klingbeil M, Kluge S, Koo H, Lacerda D, Levin MJ, Lorenzi H, Louie T, Machado CR, McCulloch R,McKenna A, Mizuno Y, Mottram JC, Nelson S, Ochaya S, Osoegawa K, Pai G, Parsons M, Pentony M, Pettersson U, Pop M, Ramirez JL, Rinta J, Robertson L, Salzberg SL, Sanchez DO, Seyler A, Sharma R, Shetty J, Simpson AJ, Sisk E, Tammi MT, Tarleton R, Teixeira S, Van Aken S, Vogt C, Ward PN, Wickstead B, Wortman J, White O, Fraser CM, Stuart KD, Andersson B 2005. The genome sequence of Trypanosoma cruzi, etiologic agent of Chagas disease. Science 309: 409-415.

Hopkins AL, Witty MJ, Nwaka S 2007. Mission possible. Nature 449: 166-169.

Jani IV, Janossy G, Brown DW, Mandy F 2002. Multiplexed immunoassays by flow cytometry for diagnosis and surveillance of infectious diseases in resource-poor settings. Lancet Infect Dis 2: 243-250.

Joos TO, Stoll D, Templin MF 2002. Miniaturised multiplexed immunoassays. Curr Opin Chem Biol 6: 76-80.

King DA, Peckham C, Waage JK, Brownlie J, Woolhouse ME 2006. Epidemiology. Infectious diseases: preparing for the future. Science 313: 1392-1393.

Krieger MA, Almeida E, Oelemann W, Lafaille JJ, Pereira JB, Krieger H, Carvalho MR, Goldenberg S 1992. Use of recombinant antigens for the accurate immunodiagnosis of Chagas' disease. $\mathrm{Am}$ J Trop Med Hyg 46: 427-434.

Lal G, Balmer P, Joseph H, Dawson M, Borrow R 2004. Development and evaluation of a tetraplex flow cytometric assay for quantitation of serum antibodies to Neisseria meningitidis serogroups A, C, Y and W-135. Clin Diagn Lab Immunol 11: 272-279.

Lee SH, Walker DR, Cregan PB, Boerma HR 2004. Comparison of four flow cytometric SNP detection assays and their use in plant improvement. Theor Appl Genet 110: 167-174.

Leon JS, Godsel LM, Wang K, Engman DM 2001. Cardiac myosin autoimmunity in acute Chagas' heart disease. Infect Immun 69: 5643-5649.

López-Antuñano FJ 1997. Quimioterapia de las infecciones producidas por Trypanosoma cruzi. Salud Publica Mex 39: 463-471.
Luan CH, Qiu S, Finley JB, Carson M, Gray RJ, Huang W, Johnson D, Tsao J, Reboul J, Vaglio P, Hill DE, Vidal M, Delucas LJ, Luo M 2004. High-throughput expression of C. elegans proteins. Genome Res 14: 2102-2110.

Martins TB, Burlingame R, von Mühlen CA, Jaskowski TD, Litwin CM, Hill HR 2004. Evaluation of multiplexed fluorescent microsphere immunoassay for detection of autoantibodies to nuclear antigens. Clin Diagn Lab Immunol 11: 1054-1059.

MSF - Médecins Sans Frontières 2008. Campaign for access to essential medicines. International meeting. New diagnostic tests are urgently needed to treat patients with Chagas disease. Rev Soc Bras Med Trop 41: 315-319.

O'Connell D 2007. Neglected diseases. Nature 449: 157-182.

O'Meara WP, Hall BF, McKenzie FE 2007. Malaria vaccine efficacy: the difficulty of detecting and diagnosing malaria. Malar J 6: 36 .

Prabhakar U, Eirikis E, Davis HM 2002. Simultaneous quantification of proinflammatory cytokines in human plasma using the LabMAP assay. J Immunol Methods 260: 207-218.

Rual JF, Hill DE, Vidal M 2004. ORFeome projects: gateway between genomics and omics. Curr Opin Chem Biol 8: 20-25.

Schmunis GA, Cruz JR 2005. Safety of the blood supply in Latin America. Clin Microbiol Rev 18: 12-29.

TDR 2007. Reporte del grupo de trabajo científico sobre la enfermedad de Chagas, 2005. Availble from: http://apps.who.int/tdr/publications/tdr-research-publications/reporte-enfermedad-chagas/ pdf/swg_chagas.pdf. [Accessed January, 5th, 2009].

Urdea M, Penny LA, Olmsted SS, Giovanni MY, Kaspar P, Shepherd A, Wilson P, Dahl CA, Buchsbaum S, Moeller G, Hay Burgess DC 2006. Requirements for high impact diagnostics in the developing world. Nature 444 (Suppl. 1): 73-79.

Vignali DAA 2000. Multiplexed particle-based flow cytometric assays. J Immunol Methods 243: 243-255.

Villa L, Morote S, Bernal O, Bulla D, Albajar-Vinas P 2007. Access to diagnosis and treatment of Chagas disease/infection in endemic and non-endemic countries in the XXI century. Mem Inst Oswaldo Cruz 102 (Suppl. I): 87-94.

Yan X, Zhong W, Tang A, Schielke EG, Hang W, Nolan JP 2005. Multiplexed flow cytometric immunoassay for influenza virus detection and differentiation. Anal Chem 77: 7673-7678. 